Title:

PLASMA PRODUCTION FROM SHOCK COMPRESSION OF CONDENSED MATTER

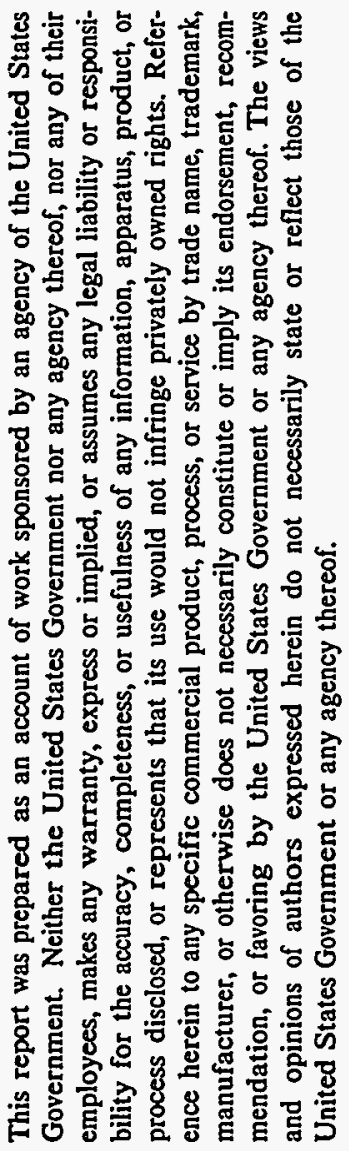

Tai-Ho Tan

Stanley P. Marsh

APS Topical Meeting on Shock Compression of Condensed Matter
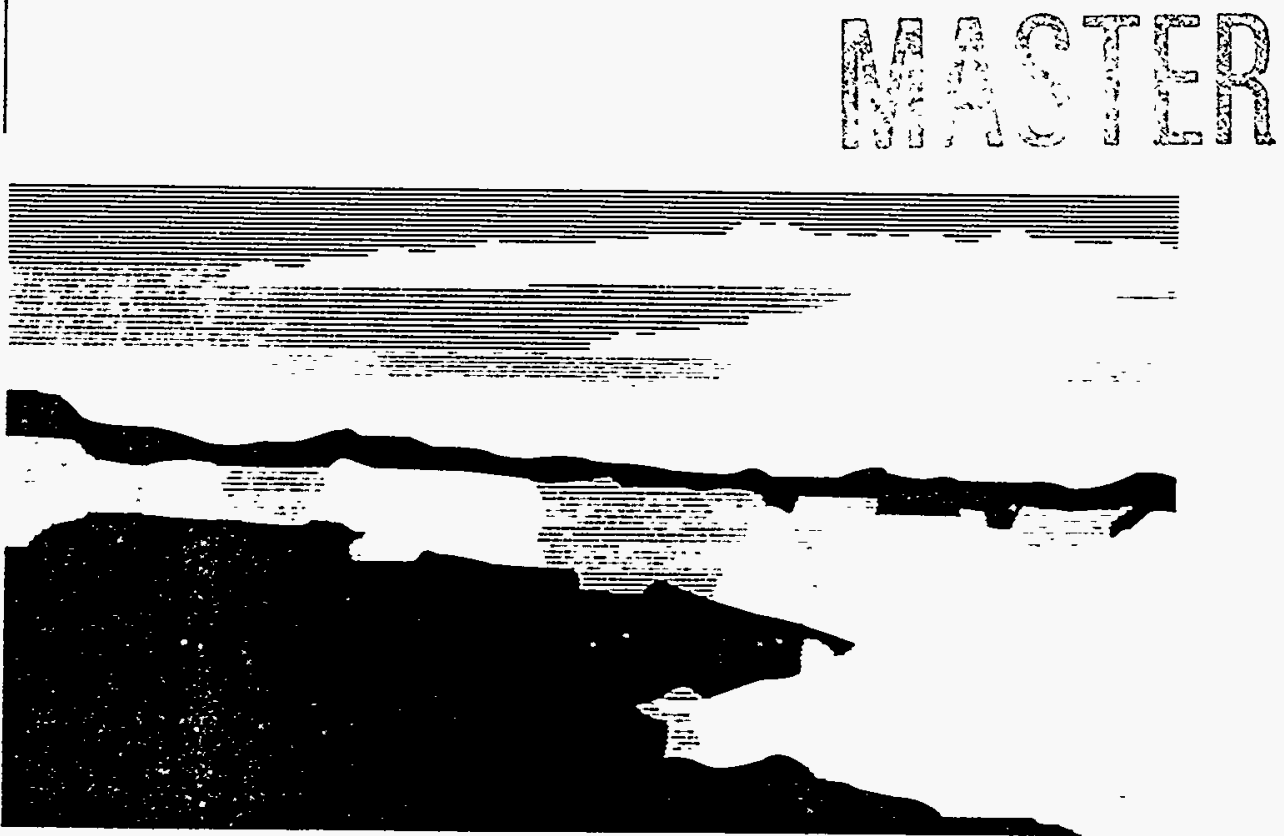

NATIONAL LABORATORY

Los Alamos National Laboratory, an affirmative action/equal opportunity employer, is operated by the University of California for the U.S. Depanment of Energy under contract W-7405-ENG-36. By acceptance of this article, the publisher recognizes that the U.S. Government retains a nonexclusive. royalty-free license to publish or reproduce the published form of this contribution, or to allow others to do so, for U.S. Government purposes. The Los Alamos National Laboratory

requests that the publisher identify this article as work performed under the auspices of the U.S. Department of Energy. 


\section{DISCLAIMER}

Portions of this document may be illegible in electronic image products. Images are produced from the best available original document. 


\title{
PLASMA PRODUCTION FROM SHOCK COMPRESSION OF CONDENSED MATTER
}

\author{
Tai-Ho Tan and Stanley P. Marsh \\ DX-15, Los Alamos National Laboratory, Los Alamos, NM, 87545
}

\begin{abstract}
The experimental investigation of HE-driven, phased, cylindrical, SS liner implosion has yielded many intercsting results. Plasma and radiation are found to be copiously produced. Plasmas with velocity up to $17 \mathrm{~cm} / \mu \mathrm{s}$ are observed. The temperature in the expansion surface reaches $8-10 \mathrm{eV}$ and stays hot for tens of microseconds. The signatures of plasma interactions with the imploding wall and the glass port are clearly identified. Finally, a cluster of cooler but still self-luminous, highdensity debris is observed to travel at $1.8 \mathrm{~cm} / \mu \mathrm{s}$. Additional experiments were carried out to study the plasma flow and reconvergence inside the liner cavity by inserting a diverting disk along the axis of implosion. Significant emission of vuv and soft $x$ rays is detected. All the experiments are guided by the calculations using the MESA 2D hydrocode and the results agree with many of the predictions.
\end{abstract}

\section{INTRODUCTION}

The study of plasma and high-frequency radiation production from a purely hydrodynamic-driven shock compression of solids is difficult because the pressure needed to reach the ionized states requires very high impacting velocity that is not easily attainable. Furthermore, when plasma is produced it is often constrained by the configuration from being observed. From our investigation of HE-driven, phased, cylindrical liner implosion we are able to mcasurc copious production of plasma and radiation. The study is motivated by the results of hydrocode calculations showing that when a hollow-core $\mathrm{HE}$ cylinder is initiated to drive a thin ss tubing, the convergence velocity can reach above $1 \mathrm{~cm} / \mu \mathrm{s}$, thus producing a $15-\mathrm{Mb}$ pressure as the density is shock compressed to $19.5 \mathrm{~g} / \mathrm{cm}^{3}$. The temperature from the heating riscs rapidly above $8 \mathrm{cV}$ and is followed by a combination of radiation and plasma emissions. The process continues for many microseconds as the phased implosion procceds along the axis, and the evolving dynamics can be observed from the open end of the cylinder. The presence of structures in the expansion front and the mainlenance of high temperature during the expansion are predicted, too. By inserting a small diameter disk along the phased implosion axis, simulation shows that the plasma flow can be perturbed and redirected to converge toward the axis. Cusps and hot spots are formed in the plume with temperature in excess of $100 \mathrm{eV}$. These are intriguing predictions that if proven valid will provide us with a means to investigate a variety of topics in interesting dynamic regimes.

In these exploratory experiments, the HE initiation simultancity and liner integrity are closely monitored to ensure that the implosion is symmetrical and free from evidence of material failure prompted by instabilitics. Radiographs of implosions are compared with those from numerical simulations. Finally, multiple diagnostics that include an extensive array of time and spatially resolved instruments are employed to measure the history and profiles of plasma expansion and radiation icmperaturc. Some of the results have been reported elsewhere. 1,2 In this paper we include samples from code calculations and a discussion of the device and experimental setup, diagnostic instrumentation, data and code comparisons.

\section{SAMPLE CALCULATIONS}

The 2D MESA Eulcrian hydrocode calculation is used to guide the design and exccution of the experiments. A few examples from the implosion of a SS liner are provided here to facilitate the discussion. Similar calculations have becn extended to other matcrials for comparison but will not bc presented. The EOS input and zone size play a 
critical role in the outcome of the simulations. These EOS values are often not totally derived from measurement, but rather from calculations based on a complicated set of physical models to cover the entire domain from solid to partially ionized gas. The end cap shown is incorporated to seal the cavity under vacuum during intense dynamic loading, a feature that makes it possible to transport plasma and soft $x$ rays unimpeded.

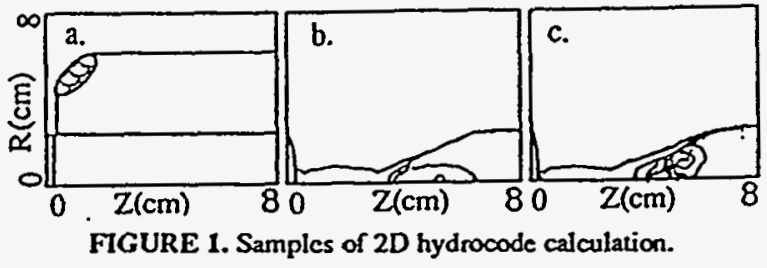

Snapshots from two different calculations are presented in Fig. 1. Time starts at zero when the corner of the HE cylinder is initiated. Relative to firing unit discharge, an additional $15.2 \mu \mathrm{s}$ burn time must be added. Figure la shows the device setup and the progression of the HE detonation wave at $2 \mu \mathrm{s}$ after the corner initiation. The liner collapses on axis at $10.3 \mu \mathrm{s}$ after reaching a velocity of $1 \mathrm{~cm} / \mu \mathrm{s}$. The maximum compressed density is calculated to be $19.5 \mathrm{~g} / \mathrm{cm}^{3}$, whilc maximum pressure and temperature are $15 \mathrm{Mb}$ and $8 \mathrm{cV}$. At this temperature the generated hol gas is partially ionized. The simulated event at $12.0 \mu \mathrm{s}$ is shown in Fig. 1b, where the expanding plasma density envelope of $10^{-3} \mathrm{~g} / \mathrm{cm}^{3}$ is moving at about $6 \mathrm{~cm} / \mu \mathrm{s}$. The fastest velocity for the very low-density component can excecd $14 \mathrm{~cm} / \mu \mathrm{s}$. Closer examination also reveals a wavy structure in the expansion front, suggesting the presence of instability. Because many details are clearly visible the dynamic profile at this time is usually chosen to compare with the radiograph. The hot gas continues to expand and manifest sign of instability at the expansion front. The temperature remains high, but may be a numerical artifact since the code does not account for heat loss. At later time, a high-density cluster of around $3 \mathrm{~g} / \mathrm{cm}^{3}$ liner debris begins to be formed and travels down the axis at about $1.8 \mathrm{~cm} / \mu \mathrm{s}$, which eventually impacts the glass port to generate a $25 \mathrm{cV}$ temperature. Figure 1c shows the convergence of plasma flow in an arrangement where a $1.25 \mathrm{~cm}$ diam $\times 0.1-\mathrm{cm}$-thick SS disk is inserted at a distance of $5 \mathrm{~cm}$. Cusps and hot spots are developed in the flow, and temperature at the convergence can reach as high as $100 \mathrm{cV}$.

\section{EXPERIMENTS}

\section{Device Description and Typical Experimental Setup}

The device description and arrangement of a typical experiment are illustrated in Fig. 2. The device, which consists of a simple, hollow core, PBX-9501 HE cylinder $(13.5 \mathrm{~cm}$ o.d $\times 5.08 \mathrm{~cm}$ i.d. $x 13.5 \mathrm{~cm}$ long), is comer initiated to implode a $5.08 \mathrm{~cm}$ o.d., $0.081-\mathrm{cm}$ thick, seamless 304 SS tubing. The ring initiation is accomplished with a single detonator by means of a thin-wall conical structure fabricated from a C2 explosives. A 0.48" $\mathrm{cm}$-thick SS sealing disk is welded to one end of the liner. The open end is glued to an expansion chamber to facilitate the ficlding of diagnostics. The chamber is made either from plastic or aluminum and may be evacuated or backfilied to any level of pressure. To measure the plasma expansion and propagation of uv and higher frequency radiation the chamber is evacuated. A plastic chamber is employed when a vacuum above a few millitorrs is adequate and it enables cameras to vicw sideways through the chamber. A metal chamber is used when higher vacuum is needed. The insert at the lower left comer shows the arrangement where a SS disk is placed along the axis to divert the plasma flow. In this configuration the HE cylinder is shortened to 6.25 $\mathrm{cm}$ in length.

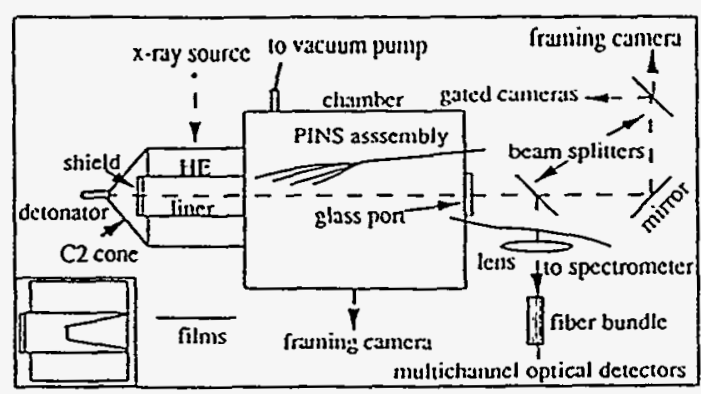

FIGURE 2. Schematic of device and experimental setup.

\section{Itemized Diagnostics and Functions}

An extensive array of diagnostic instrumentation is ficlded in cach shot, and is designed to assess the performance and to reveal temporal and spatial information that can be correlated with the simulations. Some are not shown and other are not 
fielded in every shot. Often, 8 electrical pins are placed in a collar around the $\mathrm{HE}$ to measure the initiation simultaneity. A radiograph is taken to assess the implosion symmetry and overall dynamic behavior. A time and spectrally resolved multichannel optical fiber detector system is employed to record light emission history inside the imploding cavity. These detectors with their color or neutral density filters are calibrated and arranged to cover four orders of magnitude in intensity. Sharing the view along the axis with the aid of a beam splitter are several time-resolved cameras. A gated 1024-channel spectrometer is fielded via an optical fiber to grab an interval of light emission for absolute spectrum determination, and it provides an in-situ calibration for the fiber detectors. An IMACON framing camera viewing sideways through the plastic chamber is included to record the expansion of plasma and liner debris. Optical pins were introduced once to measure the plasma expansion velocity, but tend to be intrusive to the experiment. Photoclectric diodes (XRD) are employed to measure the $u v$, vuv and soft $x$-ray cmissions.

\section{Results and Comparisons with Code Predictions}

In all the experiments the electrical pin data have consistently shown that the initiation around the corner of the HE cylinder is simultancous to within $25.5 \mathrm{~ns}$ and starts at $15.2 \mu \mathrm{s}$ after the firing discharge. All the experimental times are measured from the discharge signal. A radiograph is shown in Fig. 3. Aside from showing implosion symmetry, it also reveals a wealth of dynamic information after the initial axial collapse, which includes the size and shape of the imploding liner over its entire length, signatures of higher density plasma and jet cmissions, shock reflection into the expanding $\mathrm{HE}$ gas, and behavior of the sealing cap. The profile from the calculation ovcrlaps remarkably well with the observed features. Similar results are oblained from exposures where a disk is inserted, and shows that the disk remains in place before collision with the imploding liner.

Measurement of light emission from the cracuated cavity provides the history of the entire dynamic process for morc than $20 \mu \mathrm{s}$. Signal of a typical fiber optic channcl is shown in Fig. 4. The start at $25.5 \mu$ s corresponds exactly to the calculated time of collapse. It rises stecply to reach a peak in

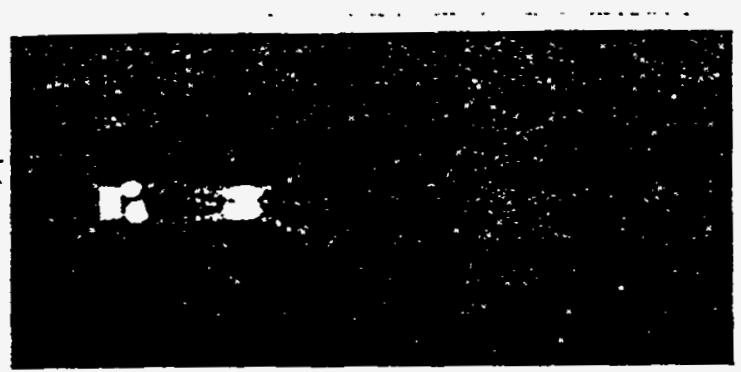

FIGURE 3. An $x$-ray radiograph taken at $27 \mu \mathrm{s}$.

about $1 \mu$ s and may be explained by plasma expansion into the imploding wall. The temperature from 26.25 to $27.25 \mu \mathrm{s}$ is estimated to be $8 \mathrm{eV}$ from intensity calibration, which is consistent with the fit to a blackbody-like spectrum from the spectrometer and in agreement with the prediction. Continuous intensity increase before the second peak may be attributed to cmissions from the expanding plasma surface, hot spots generated by instability and continuous implosion. The calculation does predict maximum temperature to remain between 8$10 \mathrm{eV}$ during the expansion. The location of the second peak at $32 \mu$ s coincides with the calculated arrival of higher density plasma at the glass port. The third peak at $36.6 \mu$ s corrclates well with the predicted final impact by the massive cluster. This is supported by the observations from the side viewing IMACON and the axially imaging rotating mirror camera. The absence of light carlicr than $25.51 \mu \mathrm{s}$ suggests that the vacuum has no leak.

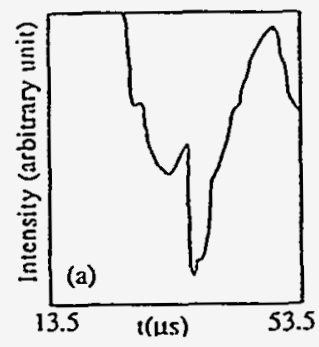

FIGURE 4. Light cmission history during implosion.

The IMACON images from the side through the plastic chamber wall were recorded at $0.5 \mu \mathrm{s}$ intervals. Using a 1-ND filter, the carly unsaturated records reveal the impact at the window by the transparent plasma. The fastest plasma velocity is thus estimated to be between $12-17 \mathrm{~cm} / \mu \mathrm{s}$, consistent with the calculation and supported by the optical pin measurements. Those images taken with 
2-ND filters clearly show the self-luminous debris entering the plastic chamber at $29.5 \mu \mathrm{s}$, advancing at a speed of about $1.9 \mathrm{~cm} / \mu \mathrm{s}$, and producing the third peak in Fig. 4, all tending to confirm the predictions.

The usefulness of ultrafast framing photography of the imploding liner cavity is compromised by its inability to accommodate the rapid variation in the object location and light emission intensity. In one setting the IMACON images were saturated because of insufficient attenuation. In another setuing, a rotating mirror camera focusing on the expansion chamber yielded no information on the collapsing cavity. However, the images of a cloud-like expansion over several microseconds corresponds closely to the debrisobserved in the IMACON. The punch-through at the port is clearly visible. Finally, a well focused snapshot taken along the axis by a fast-gated electronic camera at $1.1 \mu s$ after the liner collapsed is shown in Fig. 5. The appearance of structures in the plasma expansion implies nonuniform density and/or temperature distribution, strongly indicating the presence of turbulence and hot spots, as suggested by the simulation. Further study of this structure may shed light toward understanding the nature of plasma behaviors in a phased implosion process.

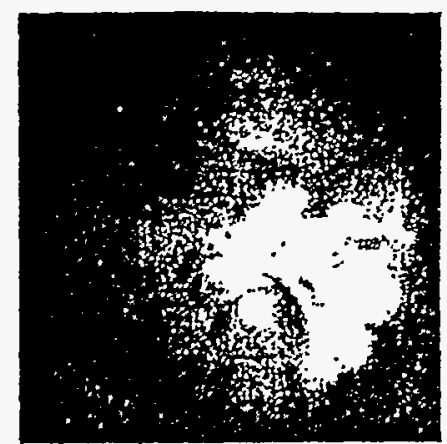

FIGURE 5. Axial vicu of expanding plasma.

In the two experiments where a disk is inserted to divert the plasma flow, a large amount of soft $x$ rays is detceted. The unfiltered channels with a threshold of $16 \mathrm{cV}$ are near saturation. However, the signal from the high-encrgy, carbon filtered channel is small, and the ratio suggests that the integrated temperature measurcd over the entire plasma plume is only about $10 \mathrm{cV}$. This is to be expected if the very high temperature hot spots represent only a very small fraction of the total area of emission. Three gated cameras triggered at different times yielded information on plasma before, during, and after flow around the disk. Interesting structures are seen in the expansion, but all the photographs were taken before the plasma plume reconverged on axis.

\section{CONCLUSIONS}

We have demonstrated that a very thin SS cylindrical liner can be driven by $H E$ to implode symmetrically and intact to very high velocity, with a substantial plasma and radiation yicld. We are pleasantly surprised to find that the data are in reasonable agrecment with many of the predictions from the numerical simulation. It appears that we may have found a powcrful tool to study interesting ultra-high pressure and temperature phenomena in the regimes that are normally investigated only with electromagnetically driven, pulsed power devices.

\section{ACKNOWLEDGEMENTS}

We are indebted to C. Findley, N. Gray, H. Oona, G. Idzorek, P. Rodriquez, S. Sterbenz, and L. Veeser for their contributions.

Rescarch supported by DOE.

\section{REFERENCES}

1. T. H. Tan and S. P. Marsh, Proccedings of the 4th International Symposium on High Pressure Dynamics, Tours, France, 1995, p. 373.

2. T. H. Tan and S. P. Marsh, Proccedings of the Intemational Symposium on Shock Waves, Pasadena, California, USA, 1995, in print. 